Relations industrielles

Industrial Relations

\title{
Loi de la convention collective
}

\section{Marcel Forget}

Volume 2, numéro 7, mars 1947

URI : https://id.erudit.org/iderudit/1023871ar

DOI : https://doi.org/10.7202/1023871ar

Aller au sommaire du numéro

Éditeur(s)

Département des relations industrielles de l'Université Laval

ISSN

0034-379X (imprimé)

1703-8138 (numérique)

Découvrir la revue

Citer cet article

Forget, M. (1947). Loi de la convention collective. Relations industrielles /

Industrial Relations, 2(7), 5-8. https://doi.org/10.7202/1023871ar

Tous droits réservés @ C Département des relations industrielles de l’Université Laval, 1947
Ce document est protégé par la loi sur le droit d'auteur. L'utilisation des services d'Érudit (y compris la reproduction) est assujettie à sa politique d'utilisation que vous pouvez consulter en ligne.

https://apropos.erudit.org/fr/usagers/politique-dutilisation/ 


\section{COLLECTIVE AGREEMENT ACT ${ }^{(1)}$}

The principle of juridical extension of collective agreements was first introduced in $19^{\prime} 4$ by the Collective Labor Agreements Extension Act; this law was completely revised in 1940 and since then has been amended annually except in 1942 and 1945.

\section{I.-Scope of the Collective Agreement Act:}

The Collective Agreement Act permits interested employers and employees, to arrange for sound industrial relations under the supervision of the State, guardian of the common good of collectivity, by establishing certain uniform working conditions which 1) standardize competition between all employers of the same category and thereby eliminate unfair competition for the signatories of private collective agreements; 2) respect the dignity of labour and provide for the needs of the worker and his family in accordance with the dictates of justice.

\section{II.-Nature of Collective Agreement:}

The collective labour agreement is a contract relative to working conditions entered into between the representatives of one or more associations of employees, on the one part, and one or more employers or representatives of one or more associations of employers, on the other part. (article 1)

\section{III.-Nature of the juridical extension}

of the collective labour agreement:

The juridical extension of the collective labour agreement is a system whereby the Lieutenant Governor in Council decrees that a collective labour agreement shall also bind, whether they are parties or not to the collective agreement, all the employees and employers of the same category within the territorial jurisdiction determined by the collective agreement. (article 2)

\section{IV.-Extent of the juridical extension \\ of collective labour agreements:}

1) Extent of professional jurisdiction:

A collective labour agreement may be extended to all employers and employees of the same trade, industry, commerce or occupation whether they are signatories or not of the said agreement, with the exception, however, of farmers, blind persons or any railway company subject to the jurisdiction of the Parliament of Canada. (article 38)

2) Extent of territorial jurisdiction:

A collective labour agreement may be extended a) to the entire province, b) to a specific region or c) to a particular municipality.

\section{V.-Procedure to obtain the juridical extension}

of a collective labour agreement:

Any party to a collective labour agreement whether representing the employers or the employees may by petition addressed to the Minister of Labour and accompanied by a duly certified copy of the collective agreement apply to the Lieutenant Governor in Council for the passing of a decree extending the effects of the said collective agreement. (articles 3 and 4). Notice of this request and the text of the collective agreement to which it relates nust be published in the Quebec Official Gazette, in a French newspaper and in an English newspaper.

Within the 30 days which follow this publication, interested parties may present objections to this agreement. I'he Minister of Labour if he deems it advisable may hold an inquiry to determine whether or not the petition or any objection made thereto is well founded. (article 5)

At the expiration of the delay mentioned above, or after having held the inquiry, as the case may be, the Minister may recommend the approval of the petition by the Lieutenant Governor in Council with such changes as are deemed expedient. He does so, however, only after first satisfying himself that:

1) the provisions of the agreement have acquired a preponderent significance and importance, that is to say that the provisions of the agreement are to the advantage of both interested employers and employees, taken as a whole;

2) the provisions of the agreement take into account the economic conditions peculiar to the various regions of the province and the competition of outside countries or other provinces.

The decree has the immediate effect of extending the collective agreement to all employers and all employees who fall under its professional and territorial jurisdiction, whether or not they were parties to the said agreement. (to be continued).

Marcel FORGE'T

(1) Authors' note.-We are presenting here in succinct form the Collective Agreement Act (R.S.Q. I94I, Chapter I63) as amended. In this way, we believe that a clear understanding of the law may be obtained. The present article is the first of a series of four articles on the subject.

\section{LOI DE LA CONVENTION COLLECTIVE}

(Suite de la page 8)

de ladite convention, à l'exception toutefois des agriculteurs, des aveugles et de toute compagnie de chemin de fer soumise à la juridiction du Parlement du Canada. (article 38)

2) champ d'application territorial:

Une convention collective de travail peut être étendue a) à toute la province, b) à une région déterminée ou c) à une ville en particulier.

V.-Procédure pour obtenir l'extension juridique

d'une convention collective de travail:

Toute partie à une convention collective de travail, qu'elle soit de l'élément patronal ou de l'élément ouvrier, peut, par requête adressée au ministre du Travail et accompagnée d'une copie dûment certifiée de la convention collective, demander au lieutenant-gouverneur en conseil l'adoption d'un décret étendant les effets de la dite convention collective. (articles 3 et 4 ) Avis de cette requête et texte de ladite convention sont publiés dans la Gazette officielle de Québec, dans un journal français et un journal anglais. Les parties intéressées peuvent dans les trente jours présenter leurs objections. Le ministre du Travail, s'il le juge à propos, fait enquête sur le bien-fondé de la requête et de toute objection formulée à l'encontre. (article 5)

A l'expiration du délai ci-dessus ou après avoir complété l'enquête précitée, le ministre peut demander au lieutenant-gouverneur en conseil d'approuver la requête, modifiée ou non sélon qu'il le juge opportun, à deux conditions:

1) que les dispositions de la convention aient acquis une signification et une importance prépondérantes, c'est-à-dire que les dispositions de la convention soient à l'avantage à la fois des employeurs et des employés intéressés pris dans leur ensemble;

2) que les dispositions de la convention tiennent compte des conditions économiques particalières aux diverses régions de la province et de la concurrence extra-provinciale ou étrangère.

Le décret déclanche l'extension juridique de la convention collective laquelle désormais s'applique à tous les employeurs et à tous les employés qui tombent sous sa juridiction professionnelle et territoriale, qu'ils aient été partie ou non partie à ladite convention. - (à suivre) 
Esquisses psychologiques III (1)

\section{L'AGENT D'AFFAIRES DU SYNDICAT}

L'agent d'affaires d'un syndicat ouvrier est une personnalite dans le domaine des relations industrielles. Il en est ainsi du directeur des relations industrielles au service d'un employeur. Ces deux types d'hommes occupent des postes stratégiques. Il en depend d'eux seuls, souvent, pour avoir, soit la paix, soit la guerre industrielles. 11 ne sera question ici que de l'agent d'aff̈aires d'un syndicat d'industrie. Peut-être revıendrons-nıus, un jour, pour parler de l'agent d'affaires d'un syndicat de métier.

L'agent d'affaures est un peu de tout: organisateur, négociateur, chef ouvrier, délégué officiel de son syndicat, homme public, éducateur. Eh oui! il doit cumuler ces multiples fonctions s'il veut répondre aux desiderata très souvent impératifs de ses ouvriers syndiqués. On nous d.ra que c'est trop exiger d'un homme. Mais tel le requiert le poste. A notre connaissance, il n'y a pas de situation plus harassante, plus exténuante que celle de notre agent d'affaires. D'ailleurs, observons la réalité des faits et nous verrons que ce type d'homme exerce des activités diverses dont il doit à tout prix s'acquitter au risque de tomber en disgrâce auprès de ses ouvriers, de l'opinion publique.

L'agent d'affaires est d'abord et avant tout un organisateur, surtout dans les débuts d'un syndicat. L'expérience nous a convaincu que le syndicat va vers l'ouvrier, non l'ouvrier vers le syndicat. L'ouvrier, règle générale, laissé à lui-même, n'est pas porté à donner une adhésion spontanée et permanente à un syndicat, quel qu'il soit. C'est la mission de l'agent d'affaires de déployer ses ressources, toutes ses ressources pour amener l'ouvrier au fait syndical et à la foi syndicale. C'est tout un art, toute une stratégie pour un agent d'affaires que de convaincre l'ouvrier qu'il y va de son intérêt vital de s'inscrire membre actif d'un syndicat.

I1 doit s'improviser homme le contacts individuels, orateur, publiciste, propagandiste. L'agent d'affaires, pour obtenir du succès, doit donc connaitre l'art de parler au public et posséder une plume dynamique et rapide. Faire du porte en porte, rédiger en vitesse des circulaires et des communiqués de presse, organiser des assemblées de syndicat et de masse et savoir les tenir d'une manière intéressante, dénicher les vrais chefs de file qui ont de l'entregent et du prestige sur leurs compagnons de travail et qui occupent, en même temps, des postes-clefs dans l'industrie, sonder l'opinion des gens à l'endroit de son syndicat, voilà la tâche colossable qui incombe à un agent d'affaires organisateur. Naturellement, il doit être intelligent, souple, aimable, dévoué, débordant de ressources. favorisé par une santé à toute épreuve, etc., etc. Et le travail prend notre homme sept jours̀ par semaine, mat:n, midi, soir, parfois même une partie de ses nuits.

Une fois son syndicat bien monté, capable de satis-

(1) N.D.L.R.-Dans cette même série, le Bulletin a déjà publié deux articles sous la signature de Gérard DION: L'employeur. volume I, nos 8 et 9 , avril et mai 1946; L'ouvrier. volume I, no 10, juin 1946. Un quatrième intitulé Le gérant de personnel par Roger McLagan de la cie Dufresne McLagan and Associates paraîtra dans le prochain numéro. faire aux exigences de la Loi des relations ouvrières, tout en demeurant organisateur, l'agent d'affaires doit cumuler le poste de négocuateur. Et c'est impératif. Én effet, les ouvriers sunissent pratiquement dans un seul et unique but: négocier une convention collective de travail. Surtout, la convention collective peut apporter des améliorations dans les conditions de travail, des augmentations ou des rajustements dans les taux de salaires, établir de bonnes relations industrielles et procurer la paix et la sécurité desirees aussi bien des ouvriers que des employeurs, des gouvernants et de l'opinion publique.

Negocier un contrat n'est pas une sinécure. C'est un art très difficile. 11 faut avoir négocié pour le savoir. Les negociatıons sont soit directes, (et elles s'élaborent dans la paix et dans un laps de temps assez court), soit forcees par les procédures de conciliation, d'arbitrage, de grève. A considerer les faits tels qu'ils sont, nous nous aemandons si nous vivons réellement en régime de civilisatıon. Les négociations directes, c'est-à-dire les ouvrierssyndiqués traitant seuls avec leur employeur, nous sembient le mode idéal de transiger une convention collective. Mais tel n est pas le cas dans les faits. Le nombre de conventions collectives négociées sans avoir recours à la conciliation ou à l'arbitrage au moins, est assez restreint. Cette constatation n'est-elle pas une preuve que nous ne sommes pas très avancés dans les relations industrielles ? A cause de ce retard de la mentalité et des moeurs relativement à la civilisation industrielle, les conflits du travail pullulent de plus en plus. C'est au stage de la négociation d'une convention collective que notre agent d'affaires traverse des quarts d'heures difficiles et qu'il est mis à l'épreuve. C'est pour lui une occasion de s'affirmer et de se placer à l'évidence. Il doit se révéler homme de caractère qui ne se laisse influencer par personne et par rien, sauf par le soucis de faire son devoir. Il doit être énergique et courtois. Il doit connaitre la législation ouvrière actuelle, notamment les principales lois, telles les lois des relations ouvrières, des différends ouvriers, des syndicats professionnels, du salaire minimum, etc. Il doit être au fait de la situation financière de la compagnie avec qui il négocie, de l'importance de l'item salaire dans le coût de production ou le prix de revient, etc. Il doit savoir lire et comprendre un bilan. Il doit être au courant du coût de la vie de la région, de la province et du pays et des salaires payés dans les industries similaires ou identiques. En un mot, au point de vue intellectuel, il ne doit pas le céder aux représentants de l'employeur. Sinon, il risque un échec ou un demi-succès. Somme toute, l'agent d'affaires doit posséder des connaissances économiques, techniques, psychologiques, légales, comptables et financières. Il le faut, parce que l'autre partie est pourvue d'un personnel qui possède ces connaissances. Le jour où les syndicats ouvriers seront en mesure de se présenter devant ieurs employeurs ou leurs représentants avec des agents d'affaires, des conseillers techniques et légaux aussi bien documentés et outillés que ceux-ci, nous assisterons alors à une période d'une véritable émancipation de la classe ouvriẹ̀re. Fait à noter (et c'est le signe des temps), toutes les centrales syndicales s'équipent de plus en plus dans ce sens.

Pendant le stage de la négociation avec l'employeur, l'agent d'affaires doit sans cesse se tenir en contact avec ses mandants, les ouvriers. C'est pour lui une question de vie. Sa tête est au jeu. Il doit informer les ouvriers sur le travail accompli, sur le résultat obtenu. Il doit exposer les chances de succès, entretenir le moral de ses hommes, souvent inquiets et impatients. C'est une des 
périodes les plus fatigantes et les plus énervantes. Car il lutte sur deux fronts à la fois: avec l'employeur pour négocier avec succès une convention collective; avec ses ouvriers qu'il doit renseigner sur les faits et sur ses propres démarches et qu'il doit surtout tenir solidaires et confiants. Aussi avons-nous connu des employeurs qui usaient de moyens dilatoires pour éterniser la signature d'une convention collective dans le but de décourager les ouvriers syndiqués, de les diviser ou de susciter de la suspicion contre leur agent d'affaires.

La convention collective enfin signée, tout n'est pas terminé, même si les deux parties sont satisfaites. Un syndicat doit toujours être vivant, avoir sans cesse un projet à réaliser. Un syndicat dont les dirigeants et les membres se reposent sur leurs lauriers est bien près de s'effondrer. Un agent d'affaires qui a réussi à négócier une belle convention collective ne peut jamais se croiser les bras et ralentir ses activités. Ce qui veut dire qu'une fois une convention collective signée, l'agent d'affaires doit en surveiller l'application. Car on ne sait jamais ! Il doit centrer son attention sur le comité de griefs. Tout grief doit avoir une solution. Le prestige de l'agent d'affaires et la confiance des ouvriers en leur syndicat résident dans le règlement des griefs. La tâche pour l'agent d'affaires, surtout quand le contrat s'est signé à la suite d'un arbitrage ou d'une grève ou encore, quand il y a rivalité syndicale dans la même industrie, s'avère des plus délicates et des plus ingrates. Au cours de la durée de la convention collective, l'agent d'affaires doit travailler à la renouveler avec nécessairement des améliorations. C'est son rôle avec le concours des officiers de son syndicat.

L'agent d'affaires est un chef ouvrier, un chef qui rayonne et en qui on a confiance. L'agent d'affaires doit être capable de lancer des mots d'ordre, d'influencer ses ouvriers syndiqués, d'être écouté, d'avoir de l'autorité. Mais jamais il ne doit être dictateur, dominateur; il ne doit pas en donner l'impression, même s'il ne l'est pas. L'agent d'affaires, réellement chef, doit inculquer un esprit de discipline et de combat en faisant coordonner les efforts de tous et de chacun vers l'obtention d'un objectif immédiat et concret et pour la réalisation, dans le temps, d'un ordre social dans lequel l'ouvrier sera intégré. L'agent d'affaires, s'il est véritablement chef ouvrier, a la mission de donner aux ouvriers l'idée de conscience et de solidarité de classe. La conscience et la solidarité de classe sont des sentiments très légitimes puisqu'il existe des classes, une classe, entre autres. la classe ouvrière. Il annartient à l'agent d'affaires d'activer et d'orienter ces deux sentiments. L'ouvrier doit penser et agir en ouvrier; il doit être convaincu que le syndicat n'est pas uniquement un organisme de coalition momentanée pour l'obtention d'un obiectif immédiat. mais surtout l'un des grands moyens à sa nortée nour l'intégrer et dans l'entreprise et dans la société. En un mot, l'agent d'affaires doit être l'architecte et l'artisan d'un monde nouveau dans le domaine des relations industrielles.

L'agent d'affaires est l'homme désigné pour représenter son syndicat là où le commandent les intérêts de ses ouvriers C'est donc un déléqué officiel. Il représente et défend ses ouvriers auprès des employeurs, des gouvernants. des commissions administratives, des tribunaux indiciaires. des organismes syndicaux supérieurs, etc. L'agent d'affaires. lorsqu'il revrésente son syndicat local. soit dans une fédération d'industrie, soit dans un conseil central, soit dans un conseil de métiers, doit lutter pour que le point de vue de son syndicat prévale tout en l'intégrant avec des horizons qui vont au-delà de sa localité ou de sa région. Notre régime industriel existe dans la province, dans le pays. L'organisation syndicale, quelle qu'elle soit, doit avoir un programme, une politique qui dépasse les limites de la région ou de la province. Cependant, l'agent d'affaires doit tenir compte des besoins et des nécessités de sa région et de sa province.

L'agent d'affaires est un homme public et un éducateur. Ses activités d'organisateur, de négociateur, de chef ouvrier, de délégué officiel en font, il va de soi, un homme public. A ce titre, il est le point de mire de tous: des membres du conseil municipal, des députés du comté, des partis politiques, des hommes d'affaires et de profession, des dirigeants des associations de toutes sortes, des chefs et des membres des syndicats rivaux, des membres du clergé, des employeurs. Surtout dans les petites villes, l'agent d'affaires est un homme qu'on observe, qu'on suit pas à pas, qu'on épie quelquefois. Ses paroles, ses écrits, ses conversations, ses gestes, ses attitudes, voire ses silences, sont remarqués, notés, commentés et critiqués, selon la gamme de sentiments qu'on entretient à son égard ou à l'endroit de son syndicat. La vie publique prend notre agent d'affaires tous les jours de l'année. Il doit sans cesse se surveiller. Car on ne lui pardonnera pas la moindre faiblesse, surtout de la part de certains employeurs qui ont intérêt à le déprécier afin de «couler» son syndicat dans l'opinion publique.

L'agent d'affaires doit consacrer beaucoup de temps et déployer ses efforts à l'éducation de son milieu ouvrier. Il doit voir à ce que chaque membre de l'exécutif s'acquitte bien et à temps de la tâche à accomplir. Il doit dénicher, parmi les ouvriers, les types qui pourront devenir de bons officiers et les former en conséquence. Il doit aider ceux qui sont en fonctions. C'est à l'agent d'affaires, le plus souvent, qu'on confie l'orientation et la direction du syndicat et des syndiqués. Il a besoin d'être dévoué, instruit, formé, doué de ressources et d'une santé parfaite. Lui qui réclame la journée de huit heures, la semaine de quarante heures, la sécurité individuelle et sociale, lui qui réclame un salaire familial et la sécurité de l'emploi, n'a pratiquement rien pour lui. Dans les faits, l'agent d'affaires est un homme qui se donne et qui sacrifie, pour une cause, un grand idéal. Il ne s'appartient plus puisqu'il appartient à un mouvement.

Joseph Pelchat

\section{Les liures}

COMITES D'ENTREPRISES - délégués du personnel, expériences étrangères, législation française. Philippe Bayart. 1 volume, 406 pages, édition du Lévrier, Montréal, 1947.

Depuis le temps ou l'associanisme ouvrier était considéré comme un délit et violemment combattu par les employeurs, il s'est produit une évolution considérable dans les relations entre les syndicats et les entreprises. De nos jours, dans plusieurs pays une collaboration institutionnelle est en train de s'établir. Dans certains cas, elle n'est que l'initiative des parties en présence; dans d'autres, elle fait l'objet de législation de la part de l'autorité publique.

Comités d'entreprises de Philippe Bayart, secrétaire du Syndicat patronal textile de Roubaix-Turcoing, veut être un tour d'horizon sur les expériences réalisées dans tous les pays où l'on a expérimenté un tel genre de collaboration patronale-ouvrière. Volume intéressant, bien documenté. qui sera très utile à tous ceux qui veulent être au courant de cette orientation nouvelle dans les relations du travail.

Gérard DION 


\section{2e CONGRÈS DES RELATIONS INDUSTRIELLES CHÂteAu frontenAc, Québec. les lundi et mardi 14 et 15 avril 1947}

\section{Pragramme}

\section{LUNDI, 14 AVRIL 1947}

Avant-midi

Président: M. Philippe Béchard, président de A. Bélanger Ltêe et directeur de l'Association Professionnelle des Industriels.

9.00 Inscription.

9.30 Allocutions de Mgr Ferdinand Vandry, P.A., V.G., Recteur de l'Université Laval et du T.R.P. G.-H. Lévesque, o.p., Doyen de la Faculté des Sciences sociales.

11.00 Préparation et technique préliminaire à la convention collective. - Forum.

M. J. O'Connell-Maher.

\section{Après-midi}

Président: M. Philippe Lessard, président de la Fédération Nationale de la Pulpe et du Papier.

2.30 Règlement des griefs au cours de lapplication de la convention. - Forum Me Donat Quimper.

4.00 Des vices généraux des conventions collectives. - Forum. Me Philippe Rousseau, c.r.

\section{MARDI, 15 AVRIL 1947}

\section{Avant-midi}

Président: M. Gérard Tremblay, Sous-ministre du Travail et Directeur du Département des relations industrielles.

9.30 Des clauses de sécurité syndicale - définition - aspect moral. - Forum. Abbé Gérard Dion.

11.00 Aspect légal des clauses de sécurité syndicale. Forum.

Me Yves Prévost, c.r.

\section{Après-midi}

Président: M. Gordon A. Ross, président de Ross Corsets Ltd. et président de la Canadian Manufacturers Association Inc., Section de la Cité de Québec.

2.30 La sécurité syndicale et l'employeur.

M. Arthur Drolet.

4.00 La sécurité syndicale et le syndicat. - Forum. M. Gérard Picard.

6.30 Dîner de clôture sous la présidence d'honneur de l'Hon. Antonio Barrette, ministre provincial du Travail.

Conférence par notre invité d'honneur: R.P. J.-T. DELOS, o.p.

Dès maintenant on peut faire son inscription au secrétariat du département des relations industrielles, Université Laval, 2 , rue de l'Université, Québec. Les frais ont été fixés à \$10.00.

\section{LOI DE LA CONVENTION COLLECTIVE ${ }^{(1)}$}

La loi qui consacre le principe de l'extension juridique des conventions collectives de travail date de 1934; elle a été remodelée entièrement une première fois en 1937, une seconde fois en 1940, et depuis lors amendée chaque année à l'exception des années 1942 et 1945.

I.-Portée de la Loi de la convention collective:

- La Loi de la convention collective permet aux parties intéressées elles-mêmes, employeurs et employés, d'aménager de saines relations professionnelles sous la surveillance de IEtat, gardien du bien commun de la collectivité, par l'uniformisation de certaines conditions de travail qui 1) égalisent les conditions de concurrence entre tous les employeurs d'une même catégorie et préviennent de la sorte une concurrence déloyale pour les signataires de conventions collectives; 2) respectent la dignité du travail et pourvoient aux besoins des salariés et de leur famille conformément aux exigences de la justice.

II.-Nature de la convention collective de travail:

La convention collective est un contrat relatif aux conditions de travail, conclu entre, d'une part, les représentants d'une ou plusieurs associations de salariés et, d'autre part, un ou plusieurs employeurs ou représentants d'une ou plusieurs associations d'employeurs. (article 1)

III.-Nature de l'extension juridique de la convention collective de travail.

L'extension juridique de la convention collective de travail est une institution par laquelle le lieutenant-gouverneur en conseil décrète qu'une convention collective de travail lie également, qu'ils soient partie ou non partie à la convention collective, tous les salariés et tous les employeurs d'une même catégorie dans la juridiction territoriale déterminée par la convention collective. (article 2)

IV.-Champ d'application de l'extension juridique de la convention collective de travail:

1) champ d'application professionnel:

Une convention collective de travail peut être étendue à tous les employeurs èt employés de tout métier, industrie, commerce ou profession, qu'ils soient signataires ou non signataires

(1) Note des auteurs.-Nous présentons ici, sous une forme succincte et qui serre de près le terte, la Loi de la Convention collećtive iS.R.Q. 1941, chapitre I63) telle qu'amendée. De cette façon, nous croyons que tous les intéressés pourront avoir de cette loi une juste et claire compréhension. Le présent article est le premier d'une série de quatre sur le sujet. - (Suite da la page 5) 